\title{
Ten-year results of a Screening Program during pregnancy for children's dental caries prophylaxis
}

\author{
Sonia Merluzzi', Tiziano Basso², Nicola Bizzaro' \\ I Laboratorio di Patologia Clinica e ${ }^{2}$ Divisione di Pediatria \\ 2 Ospedale S. Antonio, Tolmezzo (UD)
}

Key words: screening, pregnant women, early childhood caries, Streptococcus mutans, dip-slide test

\author{
Risultati di un programma decennale di Screening in gravidanza per la profilassi della carie \\ dentale infantile
}

\section{SUMMARY}

Introduction: Dental caries is a multifactorial disease with many contributing factors in the genesis of risk ( $\mathrm{I}$ ). Streptococcus mutans (SM) is a gram positive, facultative anaerobe commonly found in the human oral cavity. Described for the first time in 1924 by Clarke, is the main germ responsible for the caries disease $(5,9)$. In fact, SM produces an insoluble extracellular polysaccharide sucrose which plays an important role as a mediator of the adhesiveness, both as a cementing molecule for other microorganisms, and to create a protected site where the microorganism can proliferate $(6,9,10)$. Its presence in the plaque is not equal for all people and is closely related to sugar consumption (9). Its transmission can take place early in the life of the child through the mother's saliva $(2,3,4,8)$. The early acquisition of this organism is associated with Early Childhood Caries (ECC) and then creates a primary colonization which is hardly removed $(I, 7)$. Paying special attention to the health of women and children, this work aimed to decrease the incidence of childhood tooth decay, streamlining preventive efforts in a population at risk.

Methods: Since 1999, all women referred to our clinic in the second trimester of pregnancy or during childbirth were offered the opportunity to perform a simple test to measure the presence of SM in saliva and have been given some advice (diet, hygiene, fuoro-prophylaxis, dental visit). The sampling of saliva was performed after chewing one paraffin tablet for about I minute. For the microbiological examination the technique of dip-slide test (CTR bacteria, Ivoclar Vivadent) was used; results were classified according to semi-quantitative classes of microorganism concentration. Women were classified positive when bacterial concentration was $\geq 100000$ $\mathrm{CFU} / \mathrm{ml}$. Besides a general advice on hygiene and diet, these women were invited to undergo a prophylactic treatment with chlorhexidine, a disinfectant with bactericidal properties against SM.

Results: $84 \%$ of surveyed women adhered to the screening (29\% pregnant women, $55 \%$ in the puerperium). In the 12-year study, $2170 / 7213$ (30\%) women had a positive saliva test and were administered chlorhexidine.

Conclusions: By means of a prenatal screening involving different professionals (microbiologist, obstetriciangynecologist, pediatrician, oral-hygienist) we could assess the likelihood of vertical transmission of SM from mother to child, and give the mothers medical advice on the risk of tooth decay in children. Preliminary data on the incidence of caries in children of those women who tested positive at the screening, confirm that our program has an important role in preventing childhood tooth decay.

\section{INTRODUZIONE}

La carie dentale è una malattia multifattoriale alla cui genesi concorrono molti fattori di rischio (11). Lo Streptococcus mutans (SM) è un batterio Gram positivo e anaerobio facoltativo che comunemente si trova nella cavità orale umana (Figura 1). Descritto per la prima volta da Clarke nel 1924, è il principale germe responsabile della patologia cariosa $(5,9)$. Infatti, lo SM produce un insolubile polisaccaride extracellulare del saccarosio, che svolge un ruolo importante come mediatore dell'adesività dello SM, sia come molecola cemen- tante per altri microrganismi, sia per creare un sito protetto ove il microrganismo può proliferare (6, $9,10)$. La sua presenza nella placca batterica non è uguale per tutte le persone ed è strettamente correlata al consumo di zuccheri (9). La sua trasmissione può avvenire nei primi mesi di vita del bambino, attraverso la saliva della madre $(2,3,4,8)$. L'acquisizione precoce di questo microrganismo è associata con la Carie Infantile Precoce $(\mathrm{ECC}=$ Early Caries of Childhood) e crea una colonizzazione primaria difficile poi da modificare $(1,7)$. Nell'ambito di una particolare attenzione alla

\section{Corresponding author: Sonia Merluzzi}

Laboratorio di Patologia Clinica

Via Morgagni, 18 - 33028 Tolmezzo (UD) - Tel.: 0433 488266/488336 - Fax: 0433488697

E-mail: sonia.merluzzi@ass3.sanita.fvg.it 


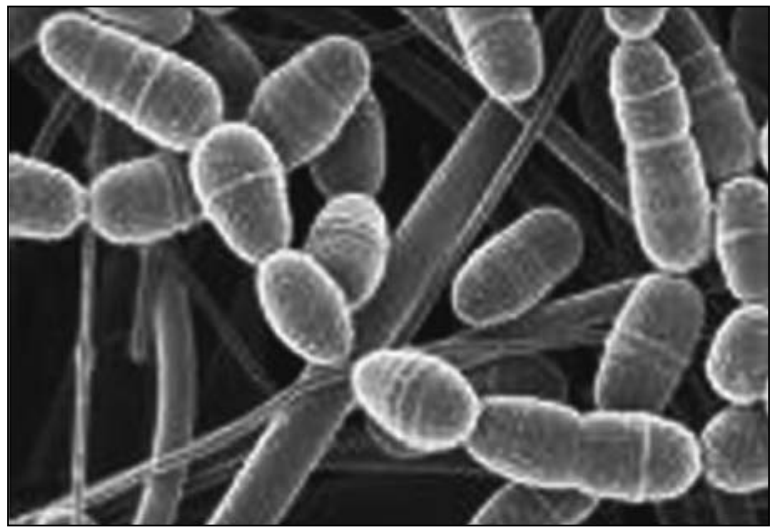

Figura I. Streptococcus mutans al microscopio elettronico a scansione.

salute della donna e del bambino si inserisce questo lavoro che ha lo scopo di diminuire l'incidenza di carie infantile, razionalizzando gli sforzi preventivi su una popolazione a rischio.

\section{METODI}

A partire dal 1999, a tutte le donne al secondo trimestre di gravidanza o in puerperio è stata offerta la possibilità di effettuare un semplice test per misurare la presenza dello SM nella saliva e sono stati dati alcuni consigli (dieta, igiene, fuoroprofilassi, visita odontoiatrica) (Figura 2).

Il campionamento di saliva è stato eseguito dopo masticazione di una compressa di paraffina per circa 1 minuto. Per l'esame microbiologico si è utilizzata la tecnica del dip-slide test (CTR bacteria, Ivoclar Vivadent) con lettura dei risultati di tipo semi-quantitativo per classi di concentrazione del microrganismo (Figura 3).

Le donne sono state classificate SM positive, quando la concentrazione batterica risultava $\geq 100000 \mathrm{UFC} / \mathrm{ml}$. Queste donne sono state invi-

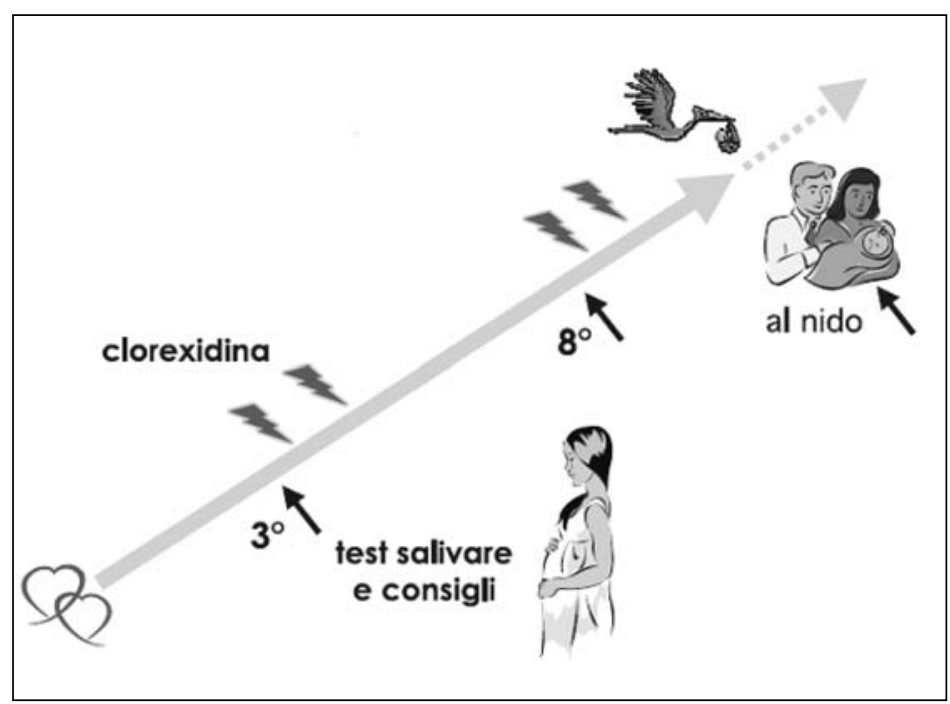

Figura II. Protocollo per lo screening dello Streotococco mutans e trattamento del rischio.

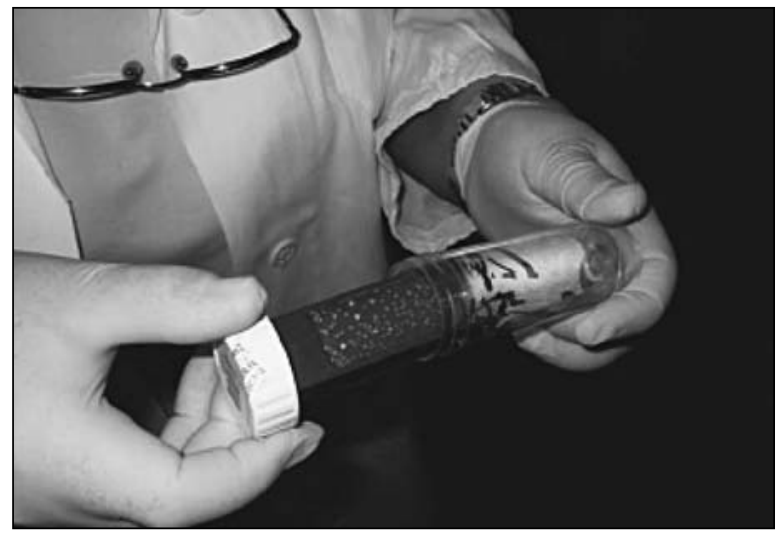

Figura III. Ricerca dello Streptococco mutans nella saliva mediante tecnica dip-slide

tate, oltre a seguire dei consigli generali di igiene e dieta, anche a sottoporsi a un trattamento con clorexidina, una sostanza disinfettante con proprietà battericida verso lo SM.

\section{RISULTATI}

L' $84 \%$ delle donne interpellate ha aderito allo screening (29\% donne gravide, 55\% nel puerperio). Delle 7213 donne studiate nei 12 anni considerati, il 30\% (2170) presentava positività al test ed è stato sottoposto a profilassi con clorexidina.

\section{CONCLUSIONI}

Nell'ambito di uno screening prenatale che vede la collaborazione di diversi professionisti (microbiologo, ginecologo-ostetrica, pediatra, dentistaigienista orale) è possibile valutare la probabilità di trasmissione verticale madre-figlio dello SM e fornire alla futura puerpera le indicazioni per ridurre il rischio di carie al figlio. Dati preliminari sull'incidenza di carie nei figli delle donne risultate positive allo screening confermano che il nostro programma ha un ruolo rilevante nella prevenzione della carie infantile.

\section{BIBLIOGRAFIA}

1. Berkowitz RJ. Causes, treatment and prevention of early childhood caries: a microbiologic perspective. J Can Dent Assoc 2003; 69: 304-7.

2. Berkowitz RJ, Jones P. Mouth-to-mouth transmission of the bacterium Streptococcus mutans between mother and child. Arch Oral Biol 1985; 30: 377-9.

3. Berkowitz RJ. Mutans streptococci: acquisition and transmission. Paediatr Dent 2006; 28: 192-8.

4. Caufield P, Cutter GR, Desanayake AP. Initial acquisition of mutans streptococci by infants: evidence for a discrete window of infectivity. J Dent Res 1993; 72: 37-45.

5. Clarke JK. On the bacterial factor in the aetiology of dental caries. Br J Exp Pathol 1924; 5: 141-6. 
6. Gibbons RJ, Houte JV. Bacterial adherence in oral microbial ecology. Annu Rev Microbiol 1975; 29: 19-44.

7. Kawashita Y, Kitamura M, Saito T. Early Childhood Caries. Int J Dent 2011; 725320.

8. Law V, Seow WK. A longitudinal controlled study of factors associated with mutans streptococci infection and caries lesion initiation in children 21 to 72 months old. Pediatr Dent 2006; 28: 58-65.
9. Loesche WJ. Role of Streptococcus mutans in human dental decay. Microbiol Rev 1986; 50: 353-80.

10. Nomura R, Naka S, Nakano K, et al. Detection of oral streptococci with collagen-binding properties in saliva specimens from mothers and their children. Int $J$ Paediatr Dent 2010; 20: 254-60.

11. Selwitz RH, Ismail AI, Pitts NB. Dental caries. Lancet 2007; 369: 51-9. 